\title{
Graphene-MoS $\mathbf{M}_{2}$ nanosheet composites as electrodes for dye sensitised solar cells
}

Peter Lynch, Umar Khan, Andrew Harvey, Iftikhar Ahmed and Jonathan N Coleman*

School of Physics, CRANN and AMBER Research Centres, Trinity College Dublin, Dublin 2, Ireland.

*colemaj@tcd.ie

\begin{abstract}
Replacing the platinum counter electrode in Dye-Senstized Solar Cells with a cheaper material has attracted much attention recently. Graphene, graphene oxide and other 2dimensional materials have received significant attention. Here we demonstrate the dependence of device parameters on the thickness of counter electrodes formed from porous, disordered arrays of liquid-exfoliated graphene nanosheets. We find device efficiency to saturate at a counter electrode thickness of $\sim 400 \mathrm{~nm}$. Such optimised counter electrodes can be improved further by adding $\mathrm{MoS}_{2}$ nanosheets to the graphene electrode. By measuring the dependence of device parameters on the composition of mixed graphene/MoS 2 electrodes, we show that adding $\sim 10 \mathrm{wt} \% \mathrm{MoS}_{2}$ nanosheets to a graphene counter electrode improves performance and can result in a cell efficiency of $\sim 95 \%$ of that achieved using a platinum electrode. This data is consistent with the $\mathrm{MoS}_{2}$ nanosheets being somewhat better catalysts than the graphene nanosheets. However, the graphene nanosheets are required to render the electrode conductive. More detailed analysis suggests the better performance of the $\mathrm{MoS}_{2}$ nanosheets to be mostly down to their smaller size.
\end{abstract}

\section{Introduction}

In 1990 the Gratzel cell or Dye-Sensitized Solar Cell (DSSC) was established as a potential electrochemical solar cell[1]. While the efficiency was relatively low, this cell type displayed considerable advantages in that it was potentially cheap to manufacture and did not require the material purity associated with silicon based solar cells. Also the cells could be made in normal ambient conditions and did not require clean room facilities[2]. Dye-Sensitized Solar Cells all employ a counter electrode which operates as a catalyst for the tri-iodide couple in the following reaction[2]: 
As with many electrochemical reactions, platinum is the most commonly used and effective catalysts for this reaction. However, for a cell type that stands out for its low cost and ease of manufacture, the platinum counter electrode has always been problematic. The high price of platinum has encouraged much research into alternative materials for use as catalytic counter electrodes in DSSCs[3].

Much of this work has focused on producing carbon-based counter electrodes. A lot of this interest is due to the high surface area and conductivity of the various forms of carbon such as activated carbon and carbon aerogels[4], carbon nanotubes[5] and graphene[6-13]. With graphene the catalytic activity is associated with active sites on the nanosheet edges[14, 15]. The simplest way to maximise the overall catalytic performance of a graphene counter electrode is to increase the number of catalytically active sites by either reducing the nanosheet size (i.e. increasing total edge length) or increasing the electrode thickness. While the second approach is relatively simple, above some critical thickness the overall catalytic activity will saturate as the process becomes limited by mass and charge transport effects[16]. In addition, from a purely economic standpoint, one would like to use the minimum graphene mass possible. The importance of thickness in these studies is often overlooked. While many of these carbon electrodes have been in excess of a micron in thickness, Kavan et al. have produced thin catalytic graphene films with high transparencies[17]. However, what is really needed is a comprehensive study on the dependence of DSSC cell performance on graphene counter electrode thickness.

In addition to purely carbon counter electrodes, a number of other materials have been studied. For example polymers, in particular PEDOT:PSS have been demonstrated as catalysts[18], as well as transition metal compounds usually with oxygen, carbon, sulfur and other chalcogenides. Many of these compounds are produced as particles with dimensions ranging from a couple of hundred nanometers to microns[19-22]. In addition, counter electrodes from arrays of two dimensional transition metal compounds are being explored such as Molybdenum and Tungsten Sulfide[23], Molybdenum Selenide[24], Tin Sulfide[25] and Cobalt Sulfide.[26] However, many of the more effective 2D catalysts are not good conductors of electricity[27]. This means the cell performance may be limited by the resistance of the counter electrode, especially for thicker electrodes. To address this problem, a number of researchers have demonstrated hybrid systems composed of a combination of conductive component, usually a 
carbon allotrope or polymer, and an electrocatalytically active material [28-33]. Of particular interest are counter electrodes formed from $\mathrm{MoS}_{2} / \mathrm{Graphene}$ hybrids. Most of the work done using $\mathrm{MoS}_{2}$ for counter electrodes has followed a synthetic route for the production of material which is then combined with pristine graphene[34, 35]. Alternatively, reduced Graphene Oxide has been used with the functional groups on Graphene Oxide acting as a site for synthesizing the $\operatorname{MoS}_{2}[36,37]$. However, in many cases these processing routes are overly complex and not in keeping with the goal of low cost, easily processed cells. This could be addressed by using a simpler route to produce $\mathrm{MoS}_{2} /$ graphene composite films. We believe Liquid Phase Exfoliation (LPE) represents such a route.

Liquid Phase Exfoliation is a very simple and scalable method for exfoliating layered crystals such as graphite, h-BN or $\mathrm{MoS}_{2}$ to give large quantities of nanosheets stably suspended, in appropriate liquids.[38-40] The resultant nanosheets have been shown to have virtually no basal plain defects introduced during the exfoliation process. The only defects are due to edge sites which tend to be electrochemically active[17, 41]. Using these methods, it is simple to produce mixed dispersions of different types of nanosheets (e.g. graphene and $\mathrm{MoS}_{2}$ ) or even nanosheets and nanotubes.[38, 42, 43] To our knowledge very little work has been done on $\mathrm{MoS}_{2}$ counter electrodes prepared by liquid phase exfoliation. While this may be due to the poor performance of $\mathrm{MoS}_{2}$ nanosheets relative to the smaller synthesized sheets and nanoparticles[44], the inherent processability of LPE nanosheets should allow significant performance enhancement via electrode optimisation.

In this work, we used LPE graphene nanosheet dispersions to produce counter electrodes with a range of thicknesses. This allowed us to identify $400 \mathrm{~nm}$ as the optimum electrode thickness. We then further improved the counter electrode performance by adding small amounts of $\mathrm{MoS}_{2}$, resulting in a DSSCs with efficiency of up to $95 \%$ of that of the equivalent platinum cell.

\section{Methods}

\section{Materials}

Graphite was purchased from. Molybdenum Disulfide, Ethyl Cellulose and Isopropyl Alcohol was purchased from Sigma Aldrich. Titania Pastes, FTO Glass, Pt Counter Electrodes and Electrolyte Materials were purchased from Dyesol.

\section{Film Production}


Dispersions of Materials were prepared by probe sonicating, using a GEX600, $48 \mathrm{~W}, 24 \mathrm{kHz}$, flat head probe, $8 \mathrm{~g}$ of material in $80 \mathrm{ml}$ of $10 \mathrm{mg} / \mathrm{ml}$ Ethyl Cellulose in Isopropyl Alcohol for 48 hours. Dispersions were then centrifuged in a Hettich Mikro 22R at $2000 \mathrm{rpm}$ for 90 minutes. Concentrations were obtained by filtration of known volumes followed by washing in isopropanol and obtaining the final mass.

Dropcast films were made by dropping known volumes of dispersion on cleaned $2 \mathrm{~cm} \times 2.5 \mathrm{~cm}$ FTO glass sheets. Different thickness were achieved by changing the deposited volume. Having to make new films for each measurement resulted in a variation in the thickness range for electrochemical testing and cell assembly. Spin coated films were fabricated by droping $200 \mu \mathrm{L}$ on a cleaned FTO glass and spinning at 1000rpm. Different thicknesses were achieved by changing concentration by dilution with Isopropyl Alcohol for thinner films or by repeating the process for thicker films. Spraycoated films were fabricated using a Harddner and Steenbeck Infinity airbrush spray system operated by a Janome JR2300N robot. The dispersion was diluted to approximately $0.1 \mathrm{mg} / \mathrm{ml}$ and sprayed onto cleaned FTO glass at $130^{\circ} \mathrm{C}$. Compositions were altered by mixing the two dispersions. Once the films were deposited by dropcasting they were heated in air at $370^{\circ} \mathrm{C}$ for 20 minutes. Thicknesses were measured using a Detak 3 Surface Profilometer.

\section{Electrochemical Measurements}

All electrochemical measurements were carried out using a Gammry 3000 Potentiostat. Electrodes were confined to an area of $1 \mathrm{~cm}^{2}$ using Surlyn $15 \mu \mathrm{m}$ sealant. Cyclic Voltagrams were measured in a three electrode setup using a Pt counter electrode and an $\mathrm{Ag} / \mathrm{AgCl}$ reference electrode at a scan rate of $50 \mathrm{mV} / \mathrm{s}$ from -0.2 to $1 \mathrm{~V}$ vs. $\mathrm{Ag} / \mathrm{AgCl}$. The electrolyte used was 0.1 $\mathrm{M} \mathrm{LiClO}_{4}, 5 \mathrm{mM} \mathrm{LiI}$, and $0.5 \mathrm{mM} \mathrm{I}_{2}$ in acetonitrle. For Linear Sweep and EIS measurements symmetrical cells were prepared using the High Performance Electrolyte provided by Dyesol. Linear Sweep scan range was from -1 to $1 \mathrm{~V}$ vs. $\mathrm{Ag} / \mathrm{AgCl}$. EIS was measured at $0.5 \mathrm{~V}$ vs. $\mathrm{Ag} / \mathrm{AgCl}$ at $10 \mathrm{mV}$ amplitude from $1 \mathrm{MHz}$ to $0.1 \mathrm{~Hz}$.

\section{Cell Production}

FTO glass was cleaned by bath sonication for $15 \mathrm{mins}$ in Decon 90 solution, deionised water, Acetone and stored in Isopropyl alcohol prior to use. FTO glass was immersed in $40 \mathrm{mM}$ solution of $\mathrm{TiCl}_{4}$ for 30 minutes at $70^{\circ} \mathrm{C}$ prior to screen printing 3 layers of approximately 4 $\mu \mathrm{m}$ of $90 \mathrm{~T}$ transparent paste followed by 1 layer of WER2-O reflector paste. These were then sintered in a furnace as described by Ito et al.[45] 
Cells were assembled as open cells separated by $15 \mathrm{~nm}$ Surlyn sealant. The working electrode was immersed in the High Performance Electrolyte provided by Dyesol while the counter electrode was clipped on to complete the cell. For comparison, the same working electrode was used for a Platinum counter electrode and a Graphene/MoS $\mathrm{S}_{2}$ counter electrode. Electrical Characteristics of the cells were obtained using a Keithly 2400 Source Meter.

\section{Results and Discussion}

We used LPE to produce suspensions of graphene nanosheets in solutions of ethyl cellulose in isopropyl alcohol (see methods). Shown in Fig 1(a) is a transmission electron microscopy (TEM) image of a typical few-layer graphene nanosheet. Statistical TEM analysis yielded a nanosheet size (L) histogram as shown in Fig 1(b) which gave an average flake size of $586 \mathrm{~nm}$. These nanosheet suspensions were formed into films by dropcasting onto glass substrates. Shown in Fig 1(c) is a scanning electron microscope image of the surface of such a film. This shows a surface morphology which is rough and porous, properties which will facilitate electrolyte penetration and access of ions to catalytic sites in the network interior. This makes these films suitable for use as counter electrodes.

In order to observe the electrochemical activity at different relative potentials, cyclic voltammetry $(\mathrm{CV})$ was performed for both a $400 \mathrm{~nm}$ thick graphene counter electrode produced by the dropcast method and, for comparison, a platinum counter electrode in an electrolyte composed of $\mathrm{I}_{3}{ }^{-} / \mathrm{I}^{-}$species in acetonitrile. The pairs of peaks between 0 and 0.4 Volts vs. $\mathrm{Ag} / \mathrm{AgCl}$ corresponds to the redox of $\mathrm{I}_{3}{ }^{-} / \mathrm{I}^{-}$while the pairs of peaks between 0.5 and $0.9 \mathrm{~V}$ vs. $\mathrm{Ag} / \mathrm{AgCl}$ are the redox couple of $\mathrm{I}_{2} / \mathrm{I}_{3}{ }^{-}[19]$. In Fig 2(a) the voltage separation between the peaks for the graphene electrode is $0.17 \mathrm{~V}$ which is smaller than the platinum separation of $0.24 \mathrm{~V}$. This provides a slight performance advantage to the graphene counter electrode. However the current densities at the graphene peaks associated with the $\mathrm{I}_{3}{ }^{-} / \mathrm{I}^{-}$couple are low relative to those of platinum, particularly for the reduction of $\mathrm{I}_{3}{ }^{-}$, which will have a negative impact on the graphene counter electrodes performance.

Dropcast Graphene electrodes of various thickness $(t=85-1100 \mathrm{~nm})$ were fabricated into symmetric cells using a high performance electrolyte provided by Dyesol and characterised using linear sweep voltagrams (Fig 2(b)). In each case, the exchange current density was extracted by fitting the data around $-1 \mathrm{~V}$ to the Tafel equation[46]. The exchange current density (Fig 2(c)) increases linearly with electrode thickness from $2 \mathrm{~mA} / \mathrm{cm}^{2}(\mathrm{t}=85 \mathrm{~nm})$ to $19 \mathrm{~mA} / \mathrm{cm}^{2}$ for a thickness of $1100 \mathrm{~nm}$. This is a reflection of the expected linear increase in the number of 
catalytic sites with electrode thickness and indicates good electrolyte penetration throughout the internal volume of the electrode. Importantly, by a thickness of $400 \mathrm{~nm}$, the exchange current density surpasses that of platinum electrodes $\left(\sim 8.5 \mathrm{~mA} / \mathrm{cm}^{2}\right)$.

We also performed electrochemical impedence spectroscopy (EIS) as shown in Fig 2(d) for electrodes of varying graphene thickness as well as Pt. In all cases, the curves were dominated by a single semicircle indicating a simplified Randel's circuit[47], allowing us to estimate the charge transfer resistance from the circle width along the $\mathrm{Z}_{\text {Real }}$ axis. The charge transfer resistances of all electrodes is plotted versus electrode thickness in figure 2(e). We find an inverse fall off with thickness from $19 \Omega(\mathrm{t}=85 \mathrm{~nm})$ to $<1 \Omega$ for a thickness of $1100 \mathrm{~nm}$. This compares to a charge transfer resistance of $\sim 6 \Omega$ for the platinum electrode. We find the charge transfer resistance to scale inversely with the exchange current density in figure 2(f) as expected from the low potential approximation to the Butler-Volmer equation[46]. This confirms the charge transfer resistance to be controlled by the number of catalytically active sites.

We used graphene films of a range of thickness as counter electrodes in DSSCs (see methods). Current density-voltage (J-V) curves for cells with different graphene electrode thicknesses are shown in Fig 3. Shown for comparison is data for a cell with a standard Pt counter electrode. It is clear from these curves that DSSC performance improves as the graphene thickness is increased (device parameters are given in table 1).

To test this thickness dependence in more detail, we fabricated cells with graphene counter electrodes which were deposited at a range of thicknesses by three different methods: dropcasting $(\mathrm{t}=60 \mathrm{~nm}-900 \mathrm{~nm})$, spin-coating $(\mathrm{t}=15 \mathrm{~nm}-360 \mathrm{~nm})$ and spray-coating $(\mathrm{t}=80 \mathrm{~nm}$ $-420 \mathrm{~nm})$. We measured the $\mathrm{J}-\mathrm{V}$ curves in all cases and extracted the short circuit density, $\mathrm{J}_{\mathrm{sc}}$, the open circuit voltage, $\mathrm{V}_{\mathrm{oc}}$, the fill factor, $\mathrm{FF}$, and the efficiency, $\mathrm{E}_{\mathrm{ff}}\left(E_{f f}=J_{s c} V_{o c} F F / P_{i n}\right.$, where $P_{\text {in }}$ is the input power). The parameters are plotted against thickness in Fig 4(a)-(d). In all cases, the values are normalised to the values measured for the Pt counter electrode. It is clear from the graphs that there is little difference between dropcasting, spin-coating and spraycoating especially at the higher range of thicknesses. For most samples it can be seen that the normalised short circuit current density of the graphene electrodes is at 80-90\% of the platinum electrode with little dependence on thickness. Increasing the thickness of the graphene layer improves the normalised open circuit voltage from $95 \%$ to $101 \%$ of platinum electrode. The most notable increase however is in the normalised fill factor which increases from $\sim 60 \%$ to 
$\sim 85 \%$ as the thickness increases from 15 to $900 \mathrm{~nm}$. Primarily because of the increase in FF, the normalised efficiency increases from $\sim 50 \%$ to $\sim 80 \%$ (relative to $\mathrm{Pt}$ ) over the thickness range. We note that there is a rapid increase in efficiency as the thickness increases to $400 \mathrm{~nm}$, after which minimal increases occur. Since the purpose of integrating graphene into the cell is to be cost competitive, we suggest that $400 \mathrm{~nm}$ is the optimum counter electrode thickness. As such this thickness was chosen for all subsequent measurements.

A number of papers have suggested $\mathrm{MoS}_{2}$ nanosheets to be effective catalysts for use in DSSC counter electrodes with evidence suggesting that they are more effective than graphene-based catalysts.[3] However, networks of $\mathrm{MoS}_{2}$ nanosheets are poor electrical conductors displaying in-plane conductivities[48] of $\sim 10^{-6} \mathrm{~S} / \mathrm{m}$ and out of plane conductivities which are three orders of magnitude lower.[49] Thus we would expect counter electrodes fabricated from networks of $\mathrm{MoS}_{2}$ nanosheets to be limited by their electrical properties. This implies that mixtures of graphene and $\mathrm{MoS}_{2}$ nanosheets could make effective counter electrodes. One would expect that while $\mathrm{MoS}_{2}$ might contribute more to the catalytic activity, graphene would dominate the charge transport. Similar strategies have recently proved successful for nanosheet-based supercapacitors, [43] lithium ion batteries[50] and hydrogen evolution catalysts[51] where addition of nanotubes has overcome resistance limitations, boosting performance.

To test this, we prepared $\mathrm{MoS}_{2}$ nanosheets by liquid phase exfoliation. Shown in figure 5 (a) is a TEM image of a typical $\mathrm{MoS}_{2}$ nanosheet. Statistical analysis yielded histograms which gave an average flake size of $156 \mathrm{~nm}$ (Fig 5(a)). Shown in figure 5(c) is an SEM of a dropcast $\mathrm{MoS}_{2}$ film on glass. Clearly the network morphology is very similar to the graphene networks.

The $\mathrm{MoS}_{2}$ and graphene suspensions were then mixed in a number of different ratios to give a set composite dispersions. These were then formed into $\mathrm{MoS}_{2} /$ graphene composite films by vacuum filtration before being transferred into glass substrates for use as counter electrodes (thickness $400 \mathrm{~nm}$ in all cases). We measured JV curves for DSSCs prepared using such composite counter electrodes with a subset shown in figure 6. It is clear from this data that the DSSC performance varies strongly with the compositional balance of $\mathrm{MoS}_{2}$ and graphene (device parameters are given in table 2).

To quantify this trend, the DSSC characteristics were extracted from the JV curves as described above, normalised to the equivalent value measured for a Pt electrode-DSSC and displayed in Fig 7 (a)-(d) as a function of graphene mass fraction. In all cases we find the $\mathrm{MoS}_{2}$ - 
only DSSCs to perform poorly with values of $\mathrm{J}_{\mathrm{sc}}, \mathrm{V}_{\mathrm{oc}}, \mathrm{FF}$, and $\mathrm{E}_{\mathrm{ff}}$ considerably lower than the equivalent values for devices with graphene-only counter electrodes. The cumulative effect of this can be seen in the normalised efficiency which was $<45 \%$ for the $\mathrm{MoS}_{2}$-only device compared to $>75 \%$ for the graphene-only device. For both $\mathrm{J}_{\mathrm{sc}}$ and $\mathrm{V}_{\mathrm{oc}}$, the normalised performance increased monotonically with graphene content in a manner roughly described by the rule of mixtures (Fig 7 (a)-(b)). However, the normalised FF behaves differently, increasing from $\sim 75 \%$ for the $\mathrm{MoS}_{2}$-only device to $\sim 115 \%$ for the counter electrode containing $90 \%$ graphene before falling to $<80 \%$ for the graphene only device (Fig 7 (c)). As a result of the behaviour of the FF, the normalised efficiency also displays non-monotonic behaviour, rising from $40 \%$ to $95 \%$ for the counter electrode containing $90 \%$ graphene before falling to $<80 \%$ for the graphene only device (Fig 7 (d)). This data clearly shows that adding small amounts of $\mathrm{MoS}_{2}$ nanosheets $(\sim 10 \mathrm{wt} \%)$ to a graphene counter electrode can give a non-trivial increase in efficiency.

We can understand these results in a phenomenological manner as follows. We assume that $\mathrm{MoS}_{2}$ is a more effective catalyst than graphene. In the simplest case the efficiency of a $\mathrm{MoS}_{2} /$ graphene mixed electrode is given by the rule of mixtures:

$$
E_{f f}=E_{f f, \text { Graphene }} M_{f}+E_{f f, M o S 2}\left(1-M_{f}\right)
$$

where $\mathrm{E}_{\mathrm{ff}, \text { graphene }}$ and $\mathrm{E}_{\mathrm{ff}, \mathrm{MoS} 2}$ are the efficiency of graphene- and $\mathrm{MoS}_{2}$-only electrodes and $\mathrm{M}_{\mathrm{f}}$ is the mass fraction of graphene in the mixed electrode. Then the efficiency should fall on adding graphene to an $\mathrm{MoS}_{2}$-only electrode. However, for electrodes dominated by $\mathrm{MoS}_{2}$, the electrode resistance will be so high as to prevent the electrode operating to its full potential. Adding graphene increases the electrode conductivity according to the percolation scaling law[43] which can be expressed approximately as

$$
\sigma \propto M_{f}^{2}
$$

(here we set the percolation threshold to zero for simplicity and take the percolation exponent as its universal value[52]). By analogy to previous results for $\mathrm{MnO}_{2} /$ nanotube based supercapacitor electrodes, addition of the graphene should increase the effectiveness of the electrode by minimising any resistance-based limitations. We proposing the efficiency of the composite electrode can be roughly modelled by modifying equation 2 by multiplying it by equation 3 to crudely account for the effect of the graphene on the electrode conductivity. Then we find: 
$E_{f f}=E_{f f 0}+k M_{f}^{2}\left[E_{f f, \text { Graphene }} M_{f}+E_{f f, M o S 2}\left(1-M_{f}\right)\right]$

Where $\mathrm{k}$ is a constant and $\mathrm{E}_{\mathrm{ff} 0}$ accounts for the fact that even in the absence of graphene, a thin layer of $\mathrm{MoS}_{2}$ near the current collector can act as an effective catalyst without resistance limitations. We have applied this expression to the data in figure $7 \mathrm{D}$, finding it to well-match the overall behaviour of the data. This analysis implies $E_{f f, M o s 2} / E_{f f, \text { Graphene }} \sim 6$ and $E_{f f, M o S 2} / E_{f f 0}$ $\sim 10$. This suggests that while the $\mathrm{MoS}_{2}$ nanosheets used here may be a far better catalyst than the graphene nanosheets, only about $10 \%$ of nanosheets in an $\mathrm{MoS}_{2}$-only electrode are close enough to the current collector to be active.

These results clearly show that the $\mathrm{MoS}_{2}$ nanosheets studied here are better catalysts for the tri-iodide reaction than the graphene sheets. In both cases, it is likely that the catalytically active sites lie on the nanosheet edge.[14, 15, 17]. This is certainly the case for $\mathrm{MoS}_{2}$ nanosheets when catalysing the hydrogen evolution reaction[41] and $\mathrm{Ni}(\mathrm{OH})_{2}$ nanosheets when catalysing the oxygen evolution reaction.[53] This means that two factors come into play, the site catalytic activity, often expressed via the turn-over frequency, and the number of sites. While the relative values of the former parameter are unknown, we can say something about the relative active site densities for $\mathrm{MoS}_{2}$ and graphene. The number of edge catalytic sites per unit volume of electrode scales inversely with nanosheet length.[51] The mean lengths of the nanosheets used here were $586 \mathrm{~nm}$ versus $156 \mathrm{~nm}$ for graphene and $\mathrm{MoS}_{2}$ respectively. This means that, assuming all sites are active and roughly equally spaced, there are about four times as many active sites per unit volume of $\mathrm{MoS}_{2}$ compared to graphene on a per unit volume basis. Moreover, the analysis of McAteer et al.[51] would suggest that the catalytic activity should scale as the turnover number, R, divided by mean nanosheet length, $<\mathrm{L}>$. Assuming that the efficiency scales roughly linearly with catalytic activity would give

$$
\frac{E_{f f, \text { MoS2 }}}{E_{f f, \text { Graphene }}} \approx \frac{R_{\text {MoS2 }}}{R_{\text {Graphene }}} \frac{\langle L\rangle_{\text {Graphene }}}{\langle L\rangle_{\text {MoS2 }}}
$$

Then, using the values given above would suggest that $R_{\text {Mos2 }} / R_{\text {Graphene }} \sim 1.5$. This implies that the $\mathrm{MoS}_{2}$ catalytic activity on a per site basis is only slightly larger than that of graphene. In fact most of the difference between $\mathrm{MoS}_{2}$ and graphene efficiencies is due to the fact that the $\mathrm{MoS}_{2}$ nanosheets used here are considerably smaller than the graphene ones. 


\section{Conclusions}

In this paper we have analysed the dependence of the performance of dye sensitised solar cells thickness on the thickness of a graphene nanosheet counter electrode, finding an optimum thickness of $400 \mathrm{~nm}$. Using this optimised thickness, we explored the effect of changing the counter electrode composition by mixing the graphene with $\mathrm{MoS}_{2}$ nanosheets. We find the performance to be optimised a composition of 90:10 graphene:MoS 2 by mass. At this composition, the efficiency was very close to that measured for a platinum counter electrode. This data is consistent with the $\mathrm{MoS}_{2}$ nanosheets being somewhat better catalysts but requiring the graphene nanosheets to render the electrode conductive. More detailed analysis suggests the better performance of the $\mathrm{MoS}_{2}$ nanosheets to be mostly down to their smaller size.

Thin film composites using Graphene and $\mathrm{MoS}_{2}$ derived from liquid phase exfoliation may represent a cheap alternative, using naturally occurring starting materials, to produce counter electrodes comparable to those produced via synthetic routes. The potential for upscaling in liquid phase exfoliation is an added benefit for driving down the price of counter electrode materials in DSSCs.

\section{Acknowledgements}

We thank Science Foundation Ireland (11/PI/1087), the European Research Council (SEMANTICS) for support.

\section{Figures}




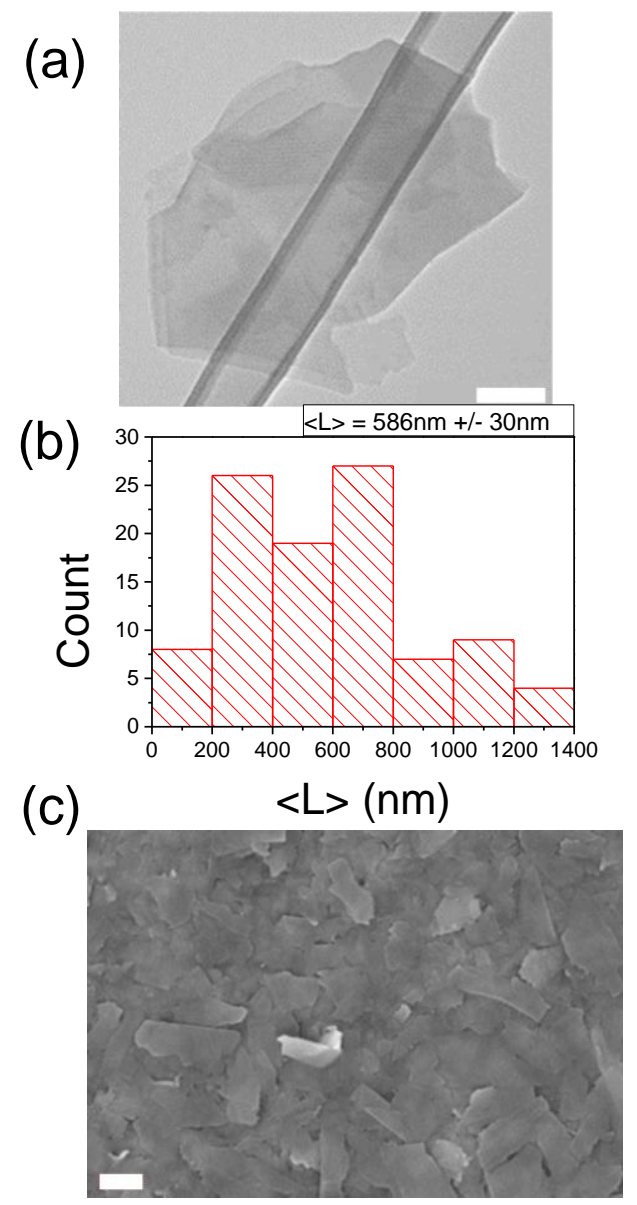

Fig 1. (a) TEM of Typical Graphene Flake. Scale Bar $=100 \mathrm{~nm}$. (b) Histogram from TEM data (c) SEM of Graphene Film. Scale bar $=500 \mathrm{~nm}$ 

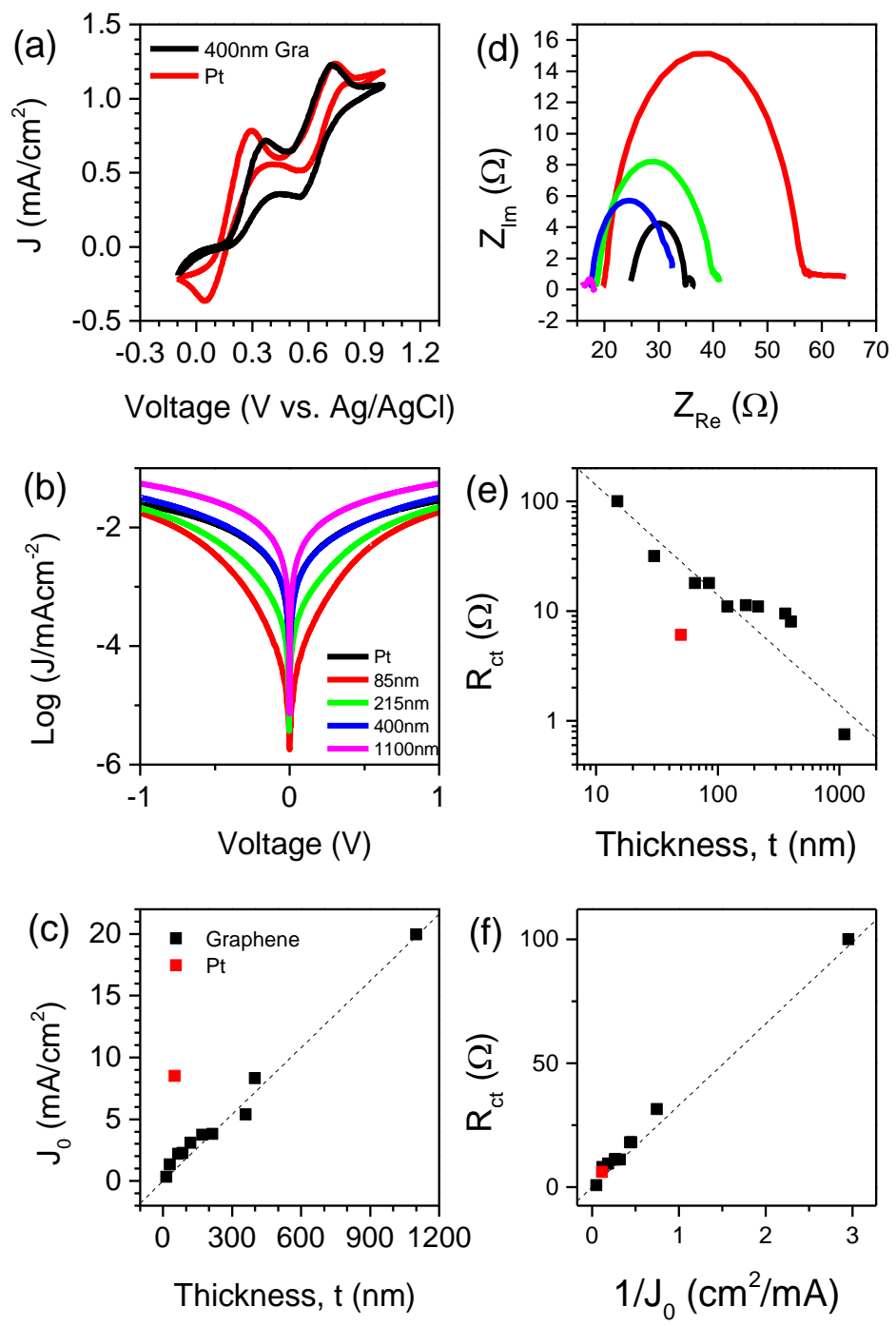

Fig 2. (a) Cyclic voltammograms measured for counter electrodes of both platinum and a 400 nm thick graphene film. (b) Tafel Plots measured for counter electrodes of platinum and dropcast graphene films of varying in thickness. (c) Exchange current density data (extracted from Tafel Plots) for graphene counter electrodes of a range of thickness. (d) Electrochemical impedance spectra measured for counter electrodes of platinum and dropcast graphene films of varying thickness. (e) Charge transfer resistance plotted versus film thickness. (f) Charge transfer resistance plotted against the inverse of the exchange current density. In (c), (e) and (f) the red square represents the datum for a Pt electrode. 


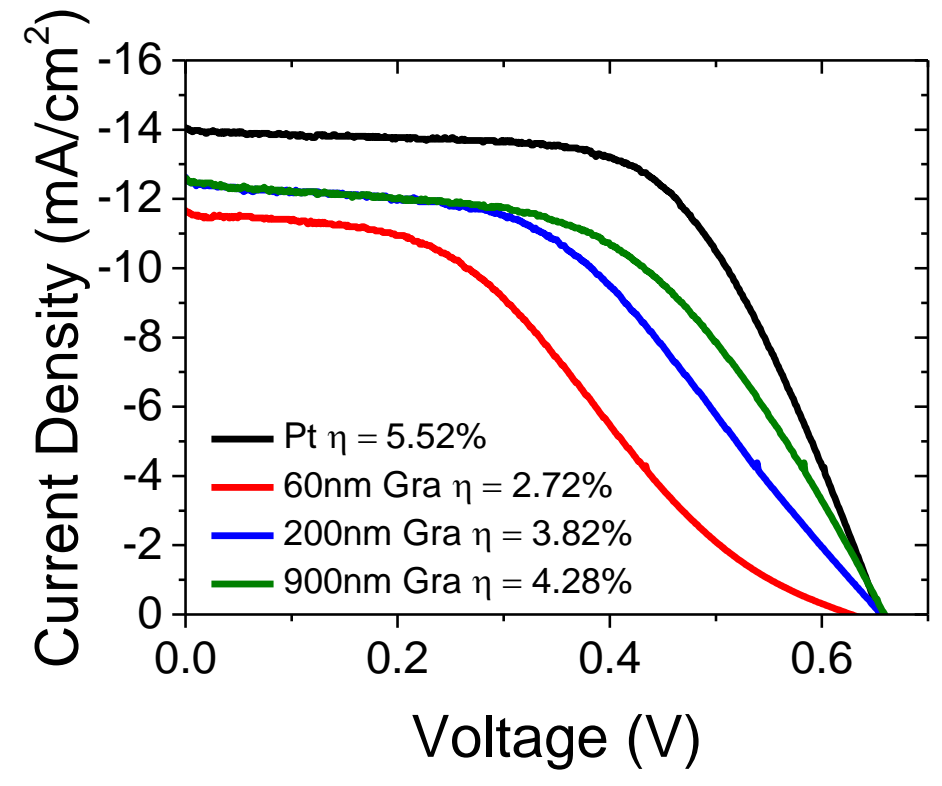

Fig. 3. IV Characteristics of DSSCs with graphene counter electrodes of different thickness. Derived parameters are shown in table 1 .
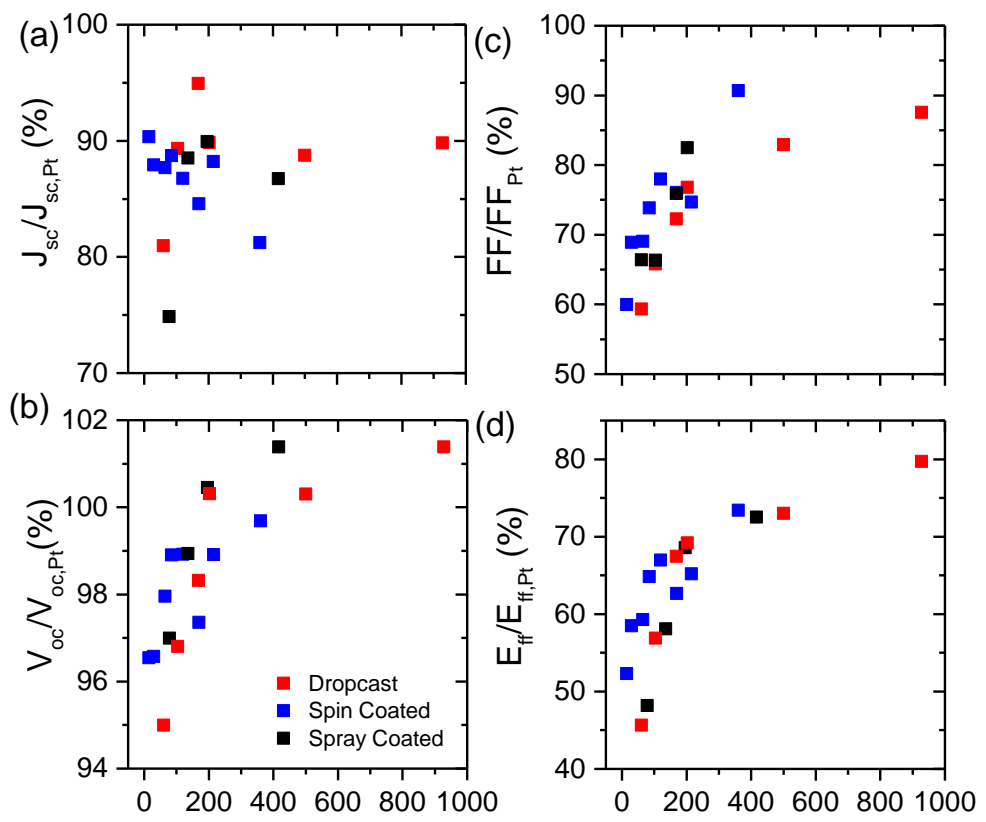

Thickness (nm) 
Fig.4 (a) Short current density, (b) open circuit voltage, (c) fill factor and (d) efficiency for DSSCs fabricated with graphene counter electrodes plotted as a function of graphene film thickness. Each parameter is expressed relative to that measured for an equivalent cell with a Platinum counter electrode.

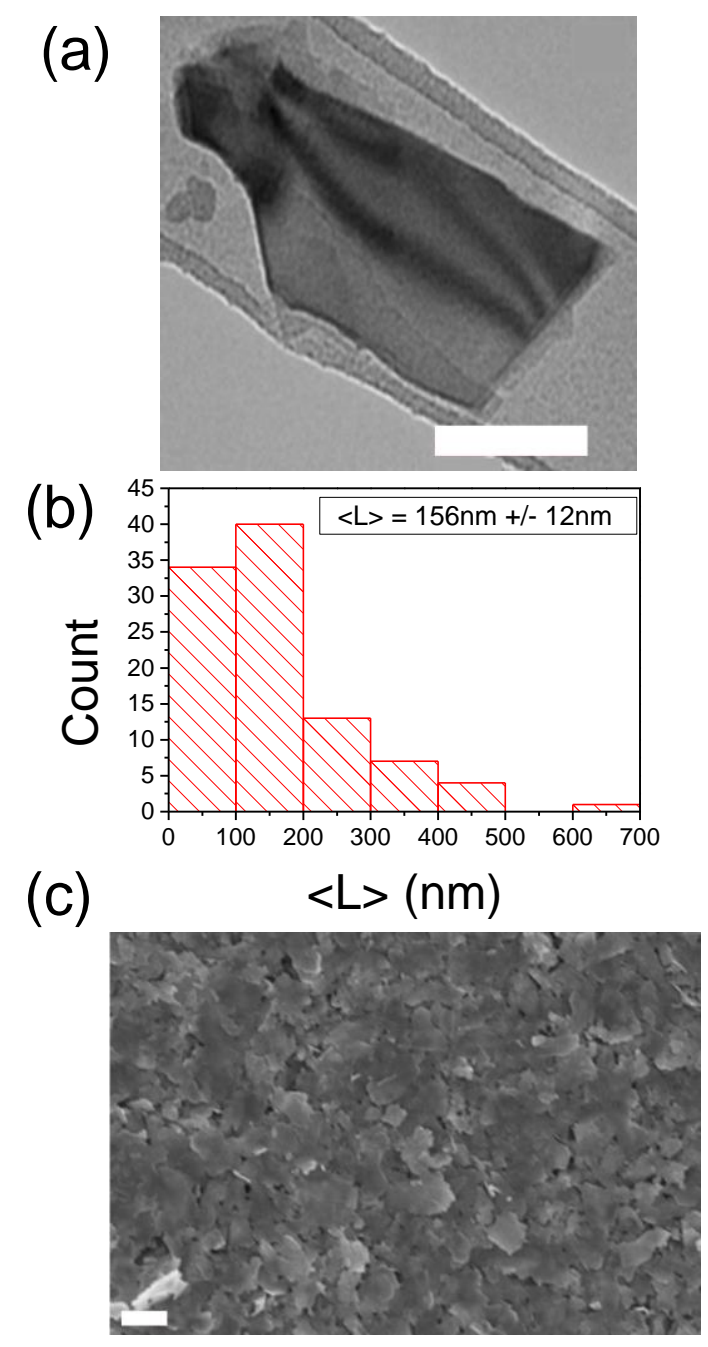

Fig 5. (a) TEM of Typical $\mathrm{MoS}_{2}$ Flake. Scale bar $=100 \mathrm{~nm}$. (b) $\mathrm{MoS}_{2}$ nanosheet size histogram extracted from TEM data. (c) SEM of $\mathrm{MoS}_{2}$ Film. Scale bar $=500 \mathrm{~nm}$ 


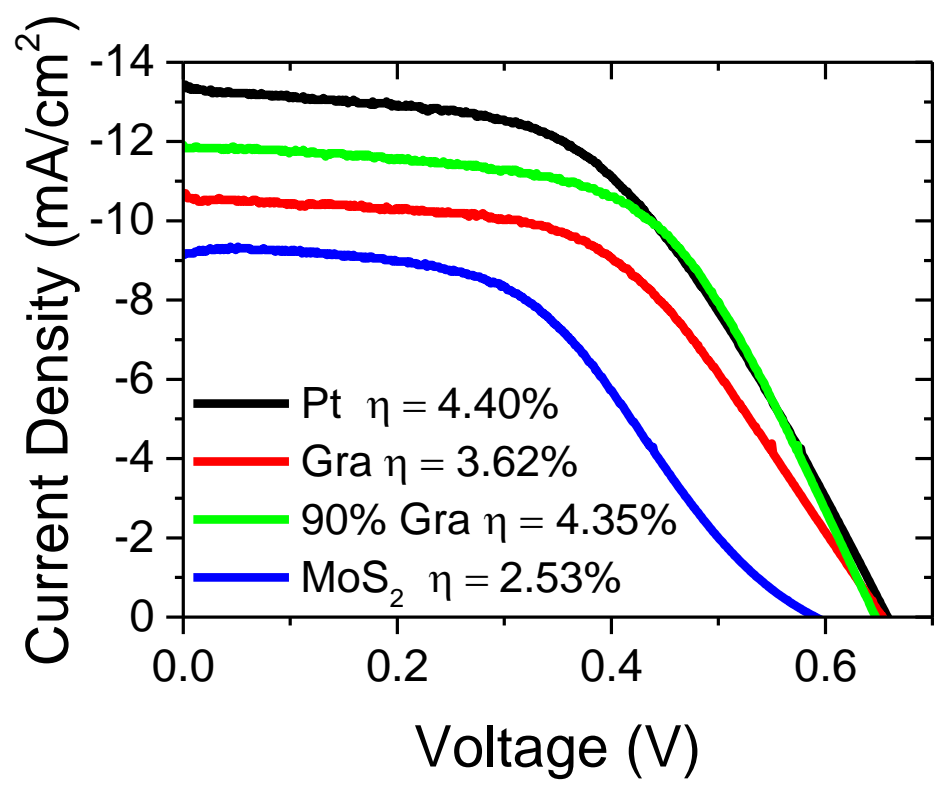

Fig. 6. IV Characteristics of DSSCs with $\mathrm{MoS}_{2}$ /graphene composite counter electrodes of different compositions. Derived parameters are shown in table 2.

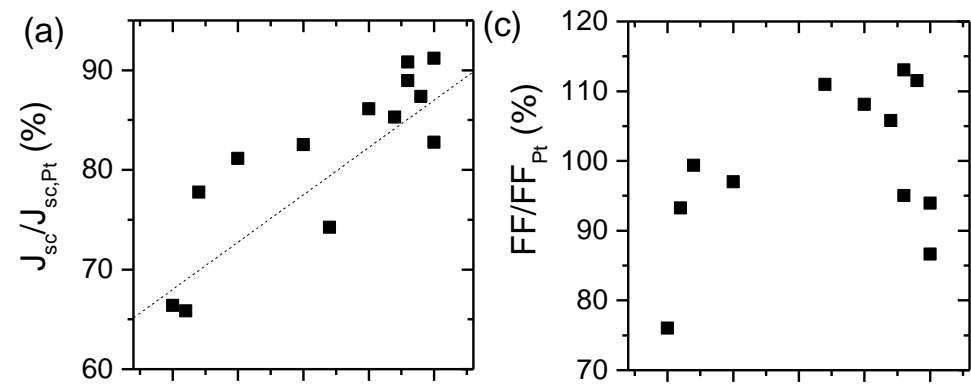

(b)

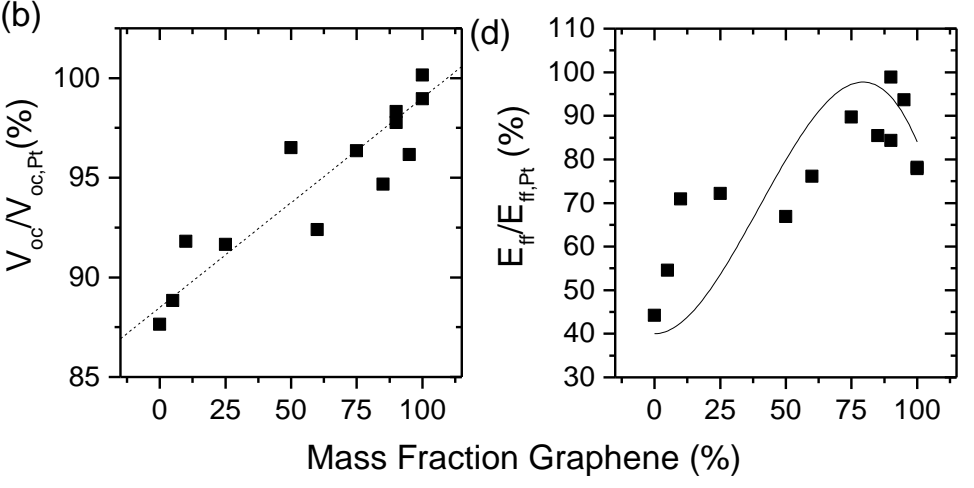


Fig.7 (a) Short current density, (b) open circuit voltage, (c) fill factor and (d) efficiency for DSSCs fabricated with $\mathrm{MoS}_{2} /$ graphene composite counter electrodes $(\mathrm{t}=400 \mathrm{~nm})$ plotted as a function of graphene mass fraction. Each parameter is expressed relative to that measured for an equivalent cell with a Platinum counter electrode. The dashed lines in (a) and (b) represent rule of mixtures type behaviour. The line in (d) is a fit to equation 4.

Table 1: Table containing cell parameters for counter electrodes of varying thickness $(t)$ - short circuit current $\left(\mathrm{J}_{\mathrm{sc}}\right)$, open circuit voltage $\left(\mathrm{V}_{\mathrm{oc}}\right)$, fill factor $(\mathrm{FF})$ and efficiency (Eff)

\begin{tabular}{|l|l|l|l|l|l|}
\hline Sample & $\mathrm{t}(\mathrm{nm})$ & $\mathrm{J}_{\mathrm{sc}}\left(\mathrm{mA} / \mathrm{cm}^{2}\right)$ & $\mathrm{V}_{\text {oc }}(\mathrm{V})$ & FF $(\%)$ & Eff $(\%)$ \\
\hline Graphene & 60 & 11.66 & 0.626 & 37.3 & 2.72 \\
\hline Graphene & 200 & 12.63 & 0.655 & 46.2 & 3.82 \\
\hline Graphene & 900 & 12.59 & 0.659 & 51.6 & 4.28 \\
\hline Platinum & n/a & 14.06 & 0.653 & 60.1 & 5.52 \\
\hline
\end{tabular}

Table 2: Table containing cell parameters for counter electrodes of varying composition - short circuit current $\left(\mathrm{J}_{\mathrm{sc}}\right)$, open circuit voltage $\left(\mathrm{V}_{\mathrm{oc}}\right)$, fill factor $(\mathrm{FF})$ and efficiency (Eff)

\begin{tabular}{|l|r|l|l|l|}
\hline Sample & $\mathrm{J}_{\mathrm{sc}}\left(\mathrm{mA} / \mathrm{cm}^{2}\right)$ & $\mathrm{V}_{\mathrm{oc}}(\mathrm{V})$ & $\mathrm{FF}(\%)$ & $\begin{array}{l}\text { Eff } \\
(\%)\end{array}$ \\
\hline Graphene & 10.7 & 0.652 & 51.9 & 3.62 \\
\hline MoS2 & 9.14 & 0.589 & 47.0 & 2.53 \\
\hline 90:10 Gra:MoS2 & 11.91 & 0.646 & 56.5 & 4.35 \\
\hline Platinum & 13.39 & 0.657 & 50.0 & 4.40 \\
\hline
\end{tabular}

\section{References}

1. O'Regan, B. and M. Gratzel, A low-cost, high-efficiency solar cell based on dye-sensitized colloidal TiO2 films. Nature, 1991. 353(6346): p. 737-740.

2. Hagfeldt, A., et al., Dye-Sensitized Solar Cells. Chemical Reviews, 2010. 110(11): p. 65956663.

3. Thomas, S., et al., A review on counter electrode materials in dye-sensitized solar cells. J. Mater. Chem. A, 2014. 2(13): p. 4474-4490. 
4. Zhao, B., et al., Flexible Counter Electrodes Based on Mesoporous Carbon Aerogel for HighPerformance Dye-Sensitized Solar Cells. The Journal of Physical Chemistry C, 2011.

115(45): p. 22615-22621.

5. Nam, J.G., et al., Enhancement of the efficiency of dye-sensitized solar cell by utilizing carbon nanotube counter electrode. Scripta Materialia, 2010. 62(3): p. 148-150.

6. $\quad$ Choi, H., et al., Graphene counter electrodes for dye-sensitized solar cells prepared by electrophoretic deposition. Journal of Materials Chemistry, 2011. 21(21): p. 7548.

7. Hung, K.-H. and H.-W. Wang, A freeze-dried graphene counter electrode enhances the performance of dye-sensitized solar cells. Thin Solid Films, 2014. 550: p. 515-520.

8. Roy-Mayhew, J.D., et al., Functionalized graphene sheets as a versatile replacement for platinum in dye-sensitized solar cells. ACS Appl Mater Interfaces, 2012. 4(5): p. 2794-800.

9. Roy-Mayhew, J.D., et al., Functionalized Graphene as a Catalytic Counter Electrode in DyeSensitized Solar Cells. ACS Nano, 2010. 4(10): p. 6203-6211.

10. Seo, H.-K., et al., New counter electrode of hot filament chemical vapor deposited graphene thin film for dye sensitized solar cell. Chemical Engineering Journal, 2013. 222: p. 464-471.

11. Wang, H. and Y.H. Hu, Graphene as a counter electrode material for dye-sensitized solar cells. Energy \& Environmental Science, 2012. 5(8): p. 8182.

12. Zhang, D.W., et al., Graphene-based counter electrode for dye-sensitized solar cells. Carbon, 2011. 49(15): p. 5382-5388.

13. Bonaccorso, F., et al., Graphene, related two-dimensional crystals, and hybrid systems for energy conversion and storage. Science, 2015. 347(6217).

14. Banks, C.E., et al., Electrocatalysis at graphite and carbon nanotube modified electrodes: edge-plane sites and tube ends are the reactive sites. Chem Commun (Camb), 2005(7): p. 829-41.

15. Yuan, W., et al., The edge- and basal-plane-specific electrochemistry of a single-layer graphene sheet. Sci Rep, 2013. 3: p. 2248.

16. Chen, D., F. Li, and A.K. Ray, Effect of mass transfer and catalyst layer thickness on photocatalytic reaction. AIChE Journal, 2000. 46(5): p. 1034-1045.

17. Kavan, L., J.H. Yum, and M. Graetzel, Optically Transparent Cathode for Dye-Sensitized Solar Cells Based on Graphene Nanoplatelets. Acs Nano, 2011. 5(1): p. 165-172.

18. Ellis, H., et al., PEDOT counter electrodes for dye-sensitized solar cells prepared by aqueous micellar electrodeposition. Electrochimica Acta, 2013. 107: p. 45-51.

19. Wu, M., et al., Economical Pt-free catalysts for counter electrodes of dye-sensitized solar cells. J Am Chem Soc, 2012. 134(7): p. 3419-28.

20. Gong, F., et al., NiSe2 as an efficient electrocatalyst for a Pt-free counter electrode of dyesensitized solar cells. Chem Commun (Camb), 2013. 49(14): p. 1437-9.

21. Guo, J., et al., Highly efficient telluride electrocatalysts for use as Pt-free counter electrodes in dye-sensitized solar cells. Chem Commun (Camb), 2013. 49(86): p. 10157-9.

22. Ji, I.A., H.M. Choi, and J.H. Bang, Metal selenide films as the counter electrode in dyesensitized solar cell. Materials Letters, 2014. 123: p. 51-54.

23. Wu, M., et al., Economical and effective sulfide catalysts for dye-sensitized solar cells as counter electrodes. Phys Chem Chem Phys, 2011. 13(43): p. 19298-301.

24. Chen, H., et al., In situ growth of a MoSe2/Mo counter electrode for high efficiency dyesensitized solar cells. Chemical Communications, 2014. 50(34): p. 4475-4477.

25. Chen, X., et al., Low-cost SnS(x) counter electrodes for dye-sensitized solar cells. Chem Commun (Camb), 2013. 49(51): p. 5793-5.

26. Tai, S.-Y., et al., Optically transparent counter electrode for dye-sensitized solar cells based on cobalt sulfide nanosheet arrays. Electrochimica Acta, 2013. 107: p. 66-70.

27. Chhowalla, M., et al., The chemistry of two-dimensional layered transition metal dichalcogenide nanosheets. Nat Chem, 2013. 5(4): p. 263-75.

28. Al-Mamun, M., et al., Pt and TCO free hybrid bilayer silver nanowire-graphene counter electrode for dye-sensitized solar cells. Chemical Physics Letters, 2013. 561-562: p. 115-119.

29. Das, S., et al., Synthesis of graphene-CoS electro-catalytic electrodes for dye sensitized solar cells. Carbon, 2012. 50(13): p. 4815-4821. 
30. Hong, W., et al., Transparent graphene/PEDOT-PSS composite films as counter electrodes of dye-sensitized solar cells. Electrochemistry Communications, 2008. 10(10): p. 1555-1558.

31. Wang, G., S. Zhuo, and W. Xing, Graphene/polyaniline nanocomposite as counter electrode of dye-sensitized solar cells. Materials Letters, 2012. 69: p. 27-29.

32. Yeh, M.-H., et al., A low-cost counter electrode of ITO glass coated with a graphene/Nafion ${ }^{\circledR}$ composite film for use in dye-sensitized solar cells. Carbon, 2012. 50(11): p. 4192-4202.

33. Zhu, G., et al., Electrophoretic deposition of reduced graphene-carbon nanotubes composite films as counter electrodes of dye-sensitized solar cells. Journal of Materials Chemistry, 2011. 21(38): p. 14869.

34. Lin, J.-Y., et al., Hydrothermal synthesis of graphene flake embedded nanosheet-like molybdenum sulfide hybrids as counter electrode catalysts for dye-sensitized solar cells. Materials Chemistry and Physics, 2013. 143(1): p. 53-59.

35. Yue, G., et al., A catalytic composite film of MoS2/graphene flake as a counter electrode for Pt-free dye-sensitized solar cells. Electrochimica Acta, 2012. 85: p. 162-168.

36. Lin, J.-Y., C.-Y. Chan, and S.-W. Chou, Electrophoretic deposition of transparent MoS2graphene nanosheet composite films as counter electrodes in dye-sensitized solar cells. Chemical Communications, 2013. 49(14): p. 1440-1442.

37. Liu, C.-J., et al., Facile synthesis of MoS2/graphene nanocomposite with high catalytic activity toward triiodide reduction in dye-sensitized solar cells. Journal of Materials Chemistry, 2012. 22(39): p. 21057.

38. Coleman, J.N., et al., Two-Dimensional Nanosheets Produced by Liquid Exfoliation of Layered Materials. Science, 2011. 331(6017): p. 568-571.

39. Hernandez, Y., et al., High-yield production of graphene by liquid-phase exfoliation of graphite. Nature Nanotechnology, 2008. 3(9): p. 563-568.

40. Nicolosi, V., et al., Liquid Exfoliation of Layered Materials. Science, 2013. 340(6139): p. 1420-+.

41. Jaramillo, T.F., et al., Identification of active edge sites for electrochemical H-2 evolution from MoS2 nanocatalysts. Science, 2007. 317(5834): p. 100-102.

42. Cunningham, G., et al., Percolation scaling in composites of exfoliated MoS2 filled with nanotubes and graphene. Nanoscale, 2012. 4(20): p. 6260-6264.

43. Higgins, T.M., et al., Effect of Percolation on the Capacitance of Supercapacitor Electrodes Prepared from Composites of Manganese Dioxide Nanoplatelets and Carbon Nanotubes. Acs Nano, 2014. 8(9): p. 9567-9579.

44. Lei, B., G.R. Li, and X.P. Gao, Morphology dependence of molybdenum disulfide transparent counter electrode in dye-sensitized solar cells. Journal of Materials Chemistry A, 2014. 2(11): p. 3919.

45. Ito, S., et al., Fabrication of thin film dye sensitized solar cells with solar to electric power conversion efficiency over 10\%. Thin Solid Films, 2008. 516(14): p. 4613-4619.

46. Bard, A.J. and L.R. Faulkner, Electrochemical Methods: Fundamentals and Applications, 2nd Edition. 2000.

47. Cooper, K.R. and M. Smith, Electrical test methods for on-line fuel cell ohmic resistance measurement. Journal of Power Sources, 2006. 160(2): p. 1088-1095.

48. Cunningham, G., et al., Large variations in both dark- and photoconductivity in nanosheet networks as nanomaterial is varied from MoS2 to WTe2. Nanoscale, 2015. 7(1): p. 198-208.

49. Cunningham, G., et al., Photoconductivity of solution-processed MoS2 films. Journal of Materials Chemistry C, 2013. 1(41): p. 6899-6904.

50. Wang, J.-Z., et al., Development of MoS2-CNT Composite Thin Film from Layered MoS2 for Lithium Batteries. Advanced Energy Materials, 2013. 3(6): p. 798-805.

51. McAteer, D., et al., Thickness Dependence and Percolation Scaling of Hydrogen Production Rate in MoS2 Nanosheet and Nanosheet-Carbon Nanotube Composite Catalytic Electrodes ACS nano online, 2015.

52. Stauffer, D. and A. Aharony, Introduction to Percolation Theory. 2nd ed. 1985, London: Taylor \& Francis.

53. Song, F. and X. Hu, Exfoliation of layered double hydroxides for enhanced oxygen evolution catalysis. Nat Commun, 2014. 5. 
